[Omitted by an oversight from the report of the December Meeting.]

\title{
A General Method of Solving the Equations of Elasticity.
}

By John Dougall, M.A.

1. In the theory of Electrostatics, or of the Newtonian potential, there exists between two systems of potentiating matter, a wellknown reciprocal relation, analytically expressed in the proposition known as Green's Theorm. By applying his theorem to the case when one of the systems is of the simplest possible character, namely, a mass concentrated at a single point, Green deduced a general method of solving the equation for the potential. The idea of a similar general method of dealing with the equations of Elasticity is due to Professor Betti, of Pisa, who has proved a reciprocal relation between two states of strain of an elastic solid, analogous to the relation in Electrostatics referred to. Following the example of Green, Betti considers what his theorem becomes when one of the states of strain belongs to one or other of certain very simple types, and obtains results which may be applied to the solution of the elastic equations. Strangely enough, however, Betti does not include among his simple types the type which we should naturally take as fundamental, namely, the strain in an infinite solid due to a force applied at a single point.

The discussion of this type of strain, from the point of view of Betti's theorem, is the object of the present paper. General theorems are reached, in which Betti's results will be seen to be included as special cases, by a method which makes physical interpretation easy.

2. In order to arrive at Betti's theorem, we start from the known principle that the potential energy of an elastic solid strained at constant temperature is a function of the strain only, i.e., is independent of the succession of steps by which the strain may be produced.

Let $(u, v, w),(\mathbf{X}, \mathbf{Y}, \mathbf{Z}),(\mathbf{F}, \mathrm{G}, \mathbf{H})$ denote respectively the displacements, components of hodily force per unit volume, and components of surface traction, in any state of equilibrium of an 
elastic solid, referred to for brevity as the state $u, v, w$. Let the same letters with accents denote the corresponding quantities in a second state of equilibrium $u^{\prime}, v^{\prime}, w^{\prime}$.

The solid may be brought from the state $u, v, w$ to the state $u^{\prime}, v^{\prime}, w^{\prime}$, by continuous passage through the states $u+n\left(u^{\prime}-u\right)$, $v+n\left(v^{\prime}-v\right), w+n\left(w^{\prime}-w\right)$, where $n$ varies from 0 to 1 . The forces required to maintain the intermediate state corresponding to a particular value of $n$ will be $\mathrm{X}+n\left(\mathrm{X}^{\prime}-\mathrm{X}\right)$, etc. The work done by the applied forces in changing the $n$ state into the $n+d n$ state will be

Volume integral of $\left\{\mathrm{X}+n\left(\mathrm{X}^{\prime}-\mathrm{X}\right)\right\}\left(u^{\prime}-u\right) d n+\ldots+\ldots$

+ Surface integral of $\left\{\mathrm{F}+n\left(\mathrm{~F}^{\prime}-\mathrm{F}\right)\right\}\left(u^{\prime}-u\right) d n+\ldots+\ldots$.

Integrating with regard to $n$ from 0 to 1 , we obtain for the total work of the applied forces in changing the state $u, v, w$ into the state $u^{\prime}, v^{\prime}, w^{\prime}$,

$$
\begin{aligned}
& \text { Volume integral of } \frac{1}{2}\left(\mathrm{X}^{\prime}+\mathrm{X}\right)\left(u^{\prime}-u\right)+\ldots+\ldots \\
&+ \text { Surface integral of } \frac{1}{2}\left(\mathrm{~F}^{\prime}+\mathrm{F}\right)\left(u^{\prime}-u\right)+\ldots+\ldots
\end{aligned}
$$

This must be equal to the excess of the potential energy in the state $u^{\prime}, v^{\prime}, w^{\prime}$, over that in the state $u, v, w$. We see that it is half the work done by the resultant of the initial and final force, acting through the increase of displacement.

In particular, if we take the state of zero potential energy to be the state of no strain, we see that the potential energy in any state is half the work done by the forces maintaining that state, acting over the displacements of that state.

Denoting the energy of any state by $\mathrm{W}(u, v, w)$ let us take for initial state $u, v, w$ and for final state $u^{\prime}-u, v^{\prime}-v, w^{\prime}-w$.

Hence $\quad \mathrm{W}\left(u^{\prime}-u, v^{\prime}-v, w^{\prime}-w\right)-\mathrm{W}(u, v, w)$

$=$ Volume integral of $\frac{1}{2} \mathrm{X}^{\prime}\left(u^{\prime}-2 u\right)+\frac{1}{2} \mathrm{Y}^{\prime}\left(v^{\prime}-2 v\right)+\frac{1}{2} \mathrm{Z}^{\prime}\left(w^{\prime}-2 w\right)$

+ Surface integral of ${ }_{2}^{1} \mathrm{~F}^{\prime}\left(u^{\prime}-2 u\right)+{ }_{2}^{1} \mathrm{G}^{\prime}\left(v^{\prime}-2 v\right)+{ }_{2}^{1} \mathrm{H}^{\prime}\left(u^{\prime}-2 u\right)$

$=\mathrm{W}\left(u^{\prime}, v^{\prime}, w\right)$

Or

$-\left\{\right.$ vol. int of $\left(\mathrm{X}^{\prime} u+\mathrm{Y}^{\prime} v+\mathrm{Z}^{\prime} w\right)+$ surf. int. of $\left.\left(\mathrm{F}^{\prime} u+\mathrm{G}^{\prime} v+\mathrm{H}^{\prime} w\right)\right\}$.

Vol. int. of $\left(\mathrm{X}^{\prime} u+\mathrm{Y}^{\prime} v+\mathrm{Z}^{\prime} w\right)+$ surf. int. of $\left(\mathrm{F}^{\prime} u+\mathrm{G}^{\prime} v+\mathrm{H}^{\prime} w\right)$

$=\mathrm{W}(u, v, w)+\mathrm{W}\left(u^{\prime}, v^{\prime}, w^{\prime}\right)-\mathrm{W}\left(u^{\prime}-u, v^{\prime}-v, w^{\prime}-w\right)$. 
But clearly $\mathrm{W}\left(u^{\prime}-u, v^{\prime}-v, w^{\prime}-w\right)=\mathrm{W}\left(u-u^{\prime}, v-v^{\prime}, w-w^{\prime}\right)$.

Hence, from symmetry

$$
\begin{aligned}
& \text { Vol. int. of }\left(\mathrm{X}^{\prime} u+\mathrm{Y}^{\prime} v+\mathrm{Z}^{\prime} w\right)+\text { surf. int. of }\left(\mathrm{F}^{\prime} u+\mathrm{G}^{\prime} v+\mathrm{H}^{\prime} w\right) \\
= & \text { Vol. int. of }\left(\mathrm{X} u^{\prime}+\mathrm{Y} v^{\prime}+\mathrm{Z} w^{\prime}\right)+\text { surf. int. of }\left(\mathrm{F} u^{\prime}+\mathrm{G} v^{\prime}+\mathrm{H} w^{\prime}\right) .
\end{aligned}
$$

This last equality is the reciprocal theorem discovered by Prof. Betti, which will be referred to simply as Betti's Theorem.

It may be stated :-If two states of equilibrium of an elastic solid be taken, then the work done by the forces of the first state acting over the displacements of the second, is equal to the work done by the forces of the second state, acting over the displacements of the first.

3. The differential equations satisfied by the components of stress at any point of an elastic solid are obtained as conditions of equilibrium of any portion of the solid. In cases where the stresses are discontinuous at a surface $S$ within the solid, these equations have to be supplemented by a surface condition.

Discontinuity in the stresses at $\mathbf{S}$ may conceivably arise from

(a) Application at the surface $\mathbf{S}$ of external force having a finite resultant per unit area.

(b) Abrupt change at $\mathbf{S}$ in the values of the elastic constants, and,

(c) So far at least as appears at first sight, abrupt change at $\mathbf{S}$ in the applied bodily force per unit volume.

Consider the equilibrium of a portion of the solid including within it part of the surface $S$, and bounded by a closed surface $S^{\prime}$.

The surface $S$ separates a region $O$ from a region $I$ of the solid; let $d \mathrm{O}, d \mathrm{I}$ denote an element of volume in these respective regions; let $l, m, n$ denote the direction cosines of the normal at any point of $\mathrm{S}$, drawn from I to $\mathrm{O}$; and $l^{\prime}, m^{\prime}, n^{\prime}$ the direction cosines of the outward normal at any point of $\mathrm{S}^{\prime}$.

Let $\mathrm{A}, \mathrm{B}, \mathrm{C}$ be the components of the force per unit area applied at $S ; X, Y, Z$ the components of the force per unit volume applied at any point of the solid. 
Resolving the forces parallel to the axis of $x$, and using the notation of Lord Kelvin and Tait for the components of stress, we have

$$
\begin{gathered}
\iint\left(l^{\mathrm{P}}+m^{\prime} \mathrm{U}+n^{\prime} \mathrm{T}\right) d \mathrm{~S}^{\prime}+\iint \mathrm{A} d \mathrm{~S}+\iiint \mathrm{X} d \mathrm{O}+\iiint \mathrm{X} d \mathrm{I}=0 \\
\text { i.e. } \iiint\left(\frac{d \mathrm{P}}{d x}+\frac{d \mathrm{U}}{d y}+\frac{d \mathrm{~T}}{d z}+\mathrm{X}\right) d \mathrm{O}+\iiint\left(\frac{d \mathrm{P}}{d x}+\frac{d \mathrm{U}}{d y}+\frac{d \mathrm{~T}}{d z}+\mathrm{X}\right) d \mathrm{I} \\
+\iint\left\{(l \mathrm{P}+m \mathrm{U}+n \mathrm{~T})_{\mathrm{o}}-(l \mathrm{P}+m \mathrm{U}+n \mathrm{~T})_{\mathrm{I}}+\mathrm{A}\right\} d \mathrm{~S}=0 .
\end{gathered}
$$

The volume integrals vanish separately from the conditions of equilibrium of a portion of the solid wholly within either region; we have then the surface condition

$$
(l \mathrm{P}+m \mathrm{U}+n \mathrm{~T})_{\mathbf{0}}-(l \mathrm{P}+m \mathrm{U}+n \mathrm{~T})_{\mathbf{1}}+\mathrm{A}=0,
$$

and similarly two other conditions of like form, which must hold at every point of $S$.

By $(l \mathrm{P}+m \mathrm{U}+n \mathrm{~T})_{0}$ is meant the value of $l \mathrm{P}+m \mathrm{U}+n \mathrm{~T}$ at $\mathrm{S}$, measured in the region $\mathrm{O}$, and similarly with $(l \mathrm{P}+m \mathrm{U}+n \mathrm{~T})_{\mathrm{I}}$.

The vanishing of the couples gives no new condition.

4. Confining ourselves now to the case of a solid, homogeneous and isotropic throughout, and subjected to no superficial internal applied force; that is, considering only, among possible sources of discontinuity of stress, discontinuity in the applied force per unit volume, we have the conditions at the surface $S$

$$
\begin{aligned}
& (l \mathrm{P}+m \mathrm{U}+n \mathrm{~T})_{\mathrm{o}}-(l \mathrm{P}+m \mathrm{U}+n \mathrm{~T})_{\mathrm{I}}=0 \\
& (l \mathrm{U}+m \mathrm{Q}+n \mathrm{~S})_{0}-(l \mathrm{U}+m \mathrm{Q}+n \mathrm{~S})_{\mathrm{I}}=0 \\
& (l \mathrm{~T}+m \mathrm{~S}+n \mathrm{R})_{0}-(l \mathrm{~T}+m \mathrm{~S}+n \mathrm{R})_{\mathrm{I}}=0 .
\end{aligned}
$$

These conditions are clearly satisfied if the tirst derivatives of $u, v, w$ are continuous at $\mathrm{S}$. This sufficient condition can be shown to be also necessary. For, in the first place, $u, v, w$ are themselves continuous, since the solid is not to be ruptured. Hence the rate of variation of $u, v, w$ per unit length in any direction lying in the surface must be continuous. 
Denoting, then, by $\left(\frac{d}{d v}\right)_{0},\left(\frac{d}{d v}\right)_{\mathrm{I}}$, rate of variation per unit length in the direction $l, m, n$ of the normal in the respective regions $O$, I, we have

and so on.

$$
\begin{aligned}
& \left(\frac{d u}{d x}\right)_{0}-\left(\frac{d u}{d x}\right)_{\mathrm{I}}=\left(l \frac{d u}{d v}\right)_{\mathrm{o}}-\left(l \frac{d u}{d v}\right)_{\mathrm{I}} \\
& \left(\frac{d u}{d y}\right)_{\mathrm{o}}-\left(\frac{d u}{d y}\right)_{\mathrm{I}}=\left(m \frac{d u}{d v}\right)_{\mathrm{O}}-\left(m \frac{d u}{d v}\right)_{\mathrm{I}}
\end{aligned}
$$

Now, using Lamé's notation for the elastic constants,

$$
\begin{gathered}
l \mathrm{P}+m \mathrm{U}+n \mathrm{~T}=l\left(\lambda \Delta+2 \mu \frac{d u}{d x}\right)+m \mu\left(\frac{d u}{d y}+\frac{d v}{d x}\right)+n \mu\left(\frac{d u}{d z}+\frac{d w}{d x}\right), \\
\text { where } \quad \Delta \equiv \frac{d u}{d x}+\frac{d v}{d y}+\frac{d w}{d z} .
\end{gathered}
$$

The above surface conditions may therefore be written

$$
\begin{aligned}
& l(\lambda+\mu)\left(l \frac{d u}{d v}+m \frac{d v}{d \nu}+n \frac{d w}{d v}\right)+\mu \frac{d u}{d \nu}=\text { same in } \mathrm{O} \text { and } \mathrm{I} \\
& m(\lambda+\mu)(\quad \text { same })+\mu \frac{d v}{d v}=\text { same in } \mathrm{O} \text { and } \mathrm{I} \\
& n(\lambda+\mu)(\text { same })+\mu \frac{d w}{d \nu}=\text { same in } \mathrm{O} \text { and } \mathrm{I} .
\end{aligned}
$$

Multiplying these equations by $l, m, n$ and adding we find

$$
l \frac{d u}{d v}+m \frac{d v}{d v}+r \frac{d w}{d v}=\text { same in } \mathrm{O} \text { and } \mathrm{I}
$$

and then, from each of the equations in turn, we find that

$$
\frac{d u}{d v}, \frac{d v}{d v}, \frac{d w}{d v} \text { are continuous. }
$$

Hence all the first derivatives of $u, v, w$ are continuous.

5. In a homogeneous isotropic solid subjected to applied force 
whose components at a point $(x, y, z)$ have any finite values $\mathrm{X}, \mathrm{Y}, \mathrm{Z}$ per unit volume, the displacements $u, v, w$ at $(x, y, z)$ satisfy the equations

$$
\begin{aligned}
& \mu \nabla^{2} u+(\lambda+\mu) \frac{d \Delta}{d x}+\mathrm{X}=0 \\
& \nabla^{2} v+(\lambda+\mu) \frac{d \Delta}{d y}+\mathrm{Y}=0 \\
& \mu \nabla^{2} v+(\lambda+\mu) \frac{d \Delta}{d z}+Z=0
\end{aligned}
$$

and we have just seen that whether $X, Y, Z$ be continuous or not, $u, v, w$ and their first derivatives are finite and continuous throughout.

Taking now any given state of strain, and any surface $\mathrm{S}$ within the solid, we may, without altering the state of strain outside $\mathbf{S}$, replace $u, v, w$ within $\mathrm{S}$ by any other functions $u^{\prime}, v^{\prime}, w^{\prime}$, which, with their first derivatives, are finite and continuous, provided

$$
u^{\prime}, \quad v^{\prime}, \quad w^{\prime}, \frac{d u^{\prime}}{d v}, \frac{d v^{\prime}}{d v}, \frac{d w^{\prime}}{d v}
$$

have at the surface $S$ the same values as

$$
u, \quad v, \quad w \quad \frac{d u}{d v}, \quad \frac{d v}{d v}, \frac{d w}{d \nu} \quad \text { respectively. }
$$

The new state of strain will require, within $S$, bodily forces given explicitly by the above equations.

As an interesting special case, $u, v, w$ may be zero at all points outside $\mathrm{S}$, provided $u^{\prime}, v^{\prime}, w^{\prime}, \frac{d u^{\prime}}{d \nu}, \frac{d v^{\prime}}{d v}, \frac{d w^{\prime}}{d \nu}$ are all zero at $S$. Thus we can find any number of systems of force within $\mathbf{S}$ which will produce absolutely no effect outside $\mathbf{S}$.

6. Again, the displacements

$$
\begin{aligned}
& \mathrm{U}_{1}=-\frac{d^{2} r}{d x^{2}}+\frac{\lambda+2 \mu}{\lambda+\mu} \frac{2}{r} \\
& \mathrm{~V}_{1}=-\frac{d^{2} r}{d x d y} \\
& \mathrm{~W}_{1}=-\frac{d^{2} r}{d x d z}
\end{aligned}
$$


where $r$ is the distance from $(x, y, z)$ to a fixed point $\left(x^{\prime}, y^{\prime}, z^{\prime}\right)$, are at once seen (observing that $\nabla^{2} r=\frac{2}{r}$ ) to satisfy the equations of equilibrium under no forces at all points except $\left(x^{\prime}, y^{\prime}, z^{\prime}\right)$, at which they become infinite.

Taking a sphere of radius $a$ about $\left(x^{\prime} y^{\prime} z^{\prime}\right)$ as centre, we may find, in the way just explained, a system of force within this sphere which will produce the above displacements at all external points.

Writing $\xi, \eta, \zeta$ for the coordinates of $(x, y, z)$ relative to $\left(x^{\prime}, y^{\prime}, z^{\prime}\right)$, the values of $U_{1}, V_{1}, W_{1}$ at the surface of the sphere are

$$
\begin{aligned}
& \mathrm{U}_{1}=\frac{\xi^{2}}{a^{3}}+\frac{\lambda+3 \mu}{\lambda+\mu} \frac{1}{a} \\
& \mathrm{~V}_{1}=\frac{\xi \eta}{a^{3}} \\
& \mathrm{~W}_{1}=\frac{\xi \xi}{a^{3}}
\end{aligned}
$$

Retaining the displacements $U_{1}, V_{1}, W_{1}$ outside the sphere, take the displacements within

$$
\begin{array}{ll}
\mathrm{U}_{0}=\frac{\xi^{2}}{a^{3}}+\frac{\lambda+3 \mu}{\lambda+\mu} \frac{1}{a}+\left(r^{2}-a^{3}\right) \mathrm{U} \\
\mathrm{V}_{0}=\frac{\xi \eta}{a^{3}} & +\left(r^{2}-a^{2}\right) \mathrm{V} \\
\mathrm{W}_{0}=\frac{\xi \zeta}{a^{3}} & +\left(r^{2}-a^{2}\right) \mathrm{W} .
\end{array}
$$

Then $U_{0}, V_{0}, W_{0}$ are equal to $U_{1}, V_{1}, W_{1}$ at the surface; in order that $\frac{d \mathrm{U}_{0}}{d r}, \frac{d \mathrm{~V}_{0}}{d r}, \frac{d \mathrm{~W}_{0}}{d r}$ should be equal to $\frac{d \mathrm{U}_{1}}{d r}, \frac{d \mathrm{~V}_{1}}{d r}, \frac{d \mathrm{~W}_{1}}{d r}$ we must have at the surface

$$
\begin{aligned}
& \mathrm{U}=-\frac{3}{2} \frac{\xi^{2}}{a^{5}}-\frac{\lambda+3 \mu}{\lambda+\mu} \frac{1}{2 a^{3}} \\
& \mathrm{~V}=-\frac{3}{2} \frac{\xi \eta}{a^{5}} \\
& \mathrm{~W}=-\frac{33}{\frac{2}{2} a^{5}}
\end{aligned}
$$

It is sufficient for our purpose to take $U, V, W$ as having these values throughout the sphere. 
The components $X_{0}, Y_{0}, Z_{0}$ of the force within the sphere can be readily calculated. They are found to be of the form

$$
\begin{aligned}
& \mathrm{X}_{0}=\mu\left(\frac{\mathrm{A}}{a^{3}}+\frac{\mathrm{B} \xi^{2}}{a^{5}}+\frac{\mathrm{C} r^{2}}{a^{5}}\right) \\
& \mathrm{Y}_{0}=\mu . \quad \frac{\mathrm{D} \xi \eta}{a^{5}} \\
& \mathrm{Z}_{0}=\mu . \quad \frac{\mathrm{D} \xi \zeta}{a^{5}},
\end{aligned}
$$

where $\mathrm{A}, \mathrm{B}, \mathrm{C}, \mathrm{D}$ are numbers.

The resultant of this system of forces is a single force parallel to the axis of $x$, and passing through $\left(x^{\prime} y^{\prime} z^{\prime}\right)$; its magnitude is

$$
\frac{8 \pi \mu(\lambda+2 \mu)}{\lambda+\mu}
$$

the reciprocal of this we shall denote by $\mathbf{M}$.

7. Take now for the displacements of a solid

$$
\begin{aligned}
& u_{1}=\mathrm{MU}_{1}=\mathrm{M}\left(-\frac{d^{2} r}{d x^{2}}+\frac{\lambda+2 \mu}{\lambda+\mu} \frac{2}{r}\right) \\
& v_{1}=\mathrm{MV}_{1}=-\mathrm{M} \frac{d^{2} r}{d x d y} \\
& w_{1}=\mathrm{MW}_{1}=-\mathrm{M} \frac{d^{2} r}{d x d z}
\end{aligned}
$$

at all points external to the sphere of radius $a$ about $\left(x^{\prime} y^{\prime} z^{\prime}\right)$; and $\mathrm{MU}_{0}, \mathrm{MV}_{0}, \mathrm{MW}_{0}$ at all internal points.

This system is maintained by the system of forces $\mathbf{M X}_{0}, \mathrm{MY}_{0}, \mathrm{MZ}_{0}$ within the sphere, the resultant of which is a unit force parallel to $\mathrm{O} x$, through the point $\left(x^{\prime} y^{\prime} z^{\prime}\right)$; along with, if the solid is bounded externally by a surface $S$ completely enclosing the sphere, surface tractions on $S$ which we shall denote by $F_{1}, G_{1}, H_{1}$, immediately calculable when the surface $S$ is known.

With a view to applying Betti's theorem, take along with this system a second system in which the external surface $S$ is held fixed by tractions $F, G, H$, and in which the displacements and components of force per unit volume at any point $(x y z)$ are $\langle u, v, w) ;(\mathrm{X}, \mathrm{Y}, \mathrm{Z})$. 
Betti's theorem gives

$$
\begin{array}{r}
\iiint\left(\mathrm{X}_{u_{1}}+\mathrm{Y} v_{1}+\mathrm{Z} w_{1}\right) d x d y d z+\iiint \mathbf{M}\left(\mathbf{X U}_{0}+\mathbf{Y} \mathrm{V}_{0}+\mathrm{ZW}_{0}\right) d \xi d \eta d \zeta \\
+\iint\left(\mathbf{F} u_{1}+\mathrm{G} v_{1}+\mathbf{H} w_{1}\right) d \mathbf{S}=\iiint \mathbf{M}\left(\mathbf{X}_{0} u+\mathbf{Y}_{0} v+\mathrm{Z}_{0} w\right) d \xi d \eta d \zeta
\end{array}
$$

the first wolume integral being taken through the space between $\mathrm{S}$ and the sphere, and the other two through the sphere.

The integral $\iiint\left(\mathrm{X} u_{1}+\mathrm{Y} v_{1}+\mathrm{Z} w_{1}\right) d \xi d \eta d \xi$ taken through the sphere, where $u_{1}, v_{1}, w_{1}$ are supposed to retain their values as given above right up to $\left(x^{\prime}, y^{\prime}, z^{\prime}\right)$, is clearly finite, since $u_{1}, v_{1}, w_{1}$ are of the order $\frac{1}{r}$ in the neighbourhood of $\left(x^{\prime} y^{\prime} z^{\prime}\right)$; adding this integral to both sides of the above equation, and transposing the second integral, we have

$$
\begin{gathered}
\iiint\left(\mathrm{X} u_{1}+\mathrm{Y} v_{1}+\mathrm{Z} w_{1}\right) d x d y d z+\iint\left(\mathrm{F} u_{1}+\mathrm{G} v_{1}+\mathrm{H} w_{1}\right) d \mathrm{~S} \\
=\iiint\left(\mathrm{X} u_{1}+\mathrm{Y}_{1}+\mathrm{Z} w_{1}\right) d \xi d \eta d \xi+\iiint \mathrm{M}\left(\mathrm{X}_{0} u+\mathrm{Y}_{0} v+\mathrm{Z}_{0} w\right) d \xi d \eta d \zeta \\
-\iiint \mathrm{M}\left(\mathrm{XU}_{0}+\mathrm{YV}_{0}+\mathrm{Z} \mathrm{W}_{0}\right) d \xi d \eta d \zeta,
\end{gathered}
$$

the first volume integral being now taken over the whole solid.

The left hand member is independent of the radius $a$; hence so also must the right. To find the value of the latter, suppose $a$ to be indefinitely diminished. The first and third of the integrals vanish in the limit; the second, from the form of $X_{0}, Y_{0}, Z_{0}$ as given in $\$ 6$, becomes

$$
u^{\prime} \times \text { Limit of } \iiint \mathrm{MX}_{0} d \xi d \eta d \zeta+\text { two similar terms }
$$

i.e. simply $u^{\prime}$, since the forces $\mathrm{MX}_{0}, \mathrm{MY}_{0}, \mathrm{MZ}_{0}$ have for resultant a unit force parallel to $\mathrm{O} x$; where $u^{\prime}$ is the value of $u$ at $\left(x^{\prime} y^{\prime} z^{\prime}\right)$.

Hence $\left.u^{\prime}=\iiint \mathrm{X} u_{1}+\mathrm{Y} v_{1}+\mathrm{Z} w_{1}\right) d x d y d z+\iint\left(\mathrm{F} u_{1}+\mathbf{G} v_{1}+\mathbf{H} w_{1}\right) d \mathbf{S}$.

If now we suppose the solid to extend to infinity, and to be 
subjected to force, continuous or discontinuous, throughout its whole extent, we have for the displacement $u^{\prime}$ at any point $\left(x^{\prime} y^{\prime} z^{\prime}\right)$, (the solid being fixed at infinity)

$$
u^{\prime}=\iiint\left(\mathrm{X} u_{1}+\mathrm{Y} v_{1}+Z w_{1}\right) d x d y d z
$$

provided $\mathrm{X}, \mathrm{Y}, \mathrm{Z}$ are such that the integral $\iint\left(\mathrm{F} u_{1}+\mathrm{G} v_{1}+\mathrm{H} w_{1}\right) d \mathrm{~S}$, taken over the surface at an infinite distance, vanishes;

and provided that the integral $\iiint\left(\mathrm{X} u_{1}+\mathrm{Y} v_{1}+\mathrm{Z} w_{1}\right) d x d y d z$ is finite.

The values of $v^{\prime}, w^{\prime}$ may be written down from symmetry.

8. Apart from the preceding application, the process of last section shows that we may apply Betti's theorem to the system $u_{1}, v_{1}, w_{1}$, supposed for mathematical purposes to extend right up to $\left(x^{\prime} y^{\prime} z^{\prime}\right)$, and that in calculating the work expressions, we must suppose in the system $u_{1}, v_{1}, w_{1}$ a unit force to exist at $\left(x^{\prime} y^{\prime} z^{\prime}\right)$.

We therefore, in the remainder of the paper, treat $u_{1} v_{1} w_{1}$, without reserve, as the displacements due to a unit force parallel to $\mathrm{O} x$ at $\left(x^{\prime} y^{\prime} z^{\prime}\right)$.

For a unit force at $\left(x^{\prime} y^{\prime} z^{\prime}\right)$, parallel to $O y$, the displacements are

$$
\begin{aligned}
& u_{2} \equiv-\mathbf{M} \frac{d^{2} r}{d y d x} \\
& v_{2} \equiv-\mathbf{M} \frac{d^{2} r}{d y^{2}}+\frac{\lambda+2 \mu}{\lambda+\mu} \frac{2 \mathrm{M}}{r} \\
& w_{2} \equiv-\mathbf{M} \frac{d^{2} r}{d y d z}
\end{aligned}
$$

and for a unit force parallel to $\mathrm{O} z$

$$
\begin{aligned}
& u_{3} \equiv-\mathbf{M} \frac{d^{2} r}{d y d x} \\
& v_{3} \equiv-\mathbf{M} \frac{d^{2} r}{d z d y} \\
& w_{3} \equiv-\mathbf{M} \frac{d^{2} r}{d z^{2}}+\frac{\lambda+2 \mu}{\lambda+\mu} \frac{2 \mathbf{M}}{r} .
\end{aligned}
$$


The symbols $u_{1}, v_{1}, w_{1} ; u_{2}, v_{2}, w_{2} ; u_{3}, v_{3}, w_{3}$ we shall use throughout as they are now defined; further, in the case when the solid is bounded by a surface $S$ at a finite distance, the necessary tractions on $\mathbf{S}$ will be denoted by

$$
\mathrm{F}_{\mathrm{i}}^{-}, \mathrm{G}_{1}, \mathrm{H}_{1} ; \mathrm{F}_{2}, \mathrm{G}_{2}, \mathrm{H}_{2} ; \mathrm{F}_{3}, \mathrm{G}_{3}, \mathbf{H}_{3} \text {. }
$$

9. From these three fundamental solutions the solutions used by Betti, referred to in $\$ 1$, may be derived as follows :

(a) Take a force $\mathrm{Q}$ parallel to $\mathrm{O} y$ at $\left(x^{\prime} y^{\prime} z^{\prime}\right)$, and a force $-\mathrm{Q}$ parallel to $\mathrm{O} y$ at $\left(x^{\prime}, y^{\prime}, z^{\prime}+h\right)$.

The displacements due to the former are $\mathrm{Q} u_{3}, \mathrm{Q} v_{2}, \mathrm{Q} w_{2}$; and to the latter $-\mathrm{Q} u_{2}^{\prime},-\mathrm{Q} v_{2}^{\prime},-\mathrm{Q} w_{2}^{\prime}$, say.

Let $\mathrm{Q}$ be increased and $h$ diminished indefinitely, so that $\mathrm{Q} h$ remains finite and equal to $\frac{1}{2}$.

The $x$-displacement due to the combination is $Q\left(u_{2}-u_{2}^{\prime}\right)$, or

$$
-\mathrm{Q} h \frac{u_{2}^{\prime}-u_{2}}{h}, \quad \text { which in the limit }=-\frac{1}{2} \frac{d u_{2}}{d z^{\prime}} \text { or } \frac{1}{2} \frac{d u_{2}}{d z} \text {. }
$$

The displacements are, therefore, $\frac{1}{2} \frac{d u_{\mathrm{n}}}{d z}, \frac{1}{2} \frac{d v_{2}}{d z}, \frac{1}{2} \frac{d w_{2}}{d z}$.

The resultant of the forces applied to the element at $\left(x^{\prime} y^{\prime} z^{\prime}\right)$ is a couple of moment $\frac{1}{2}$ in the $y z$ plane ;

the work done by these forces acting through any system of displacements $u, v, w$ is $-\frac{1}{2}\left(\frac{d v}{d z}\right)^{\prime}$, the value of $-\frac{1}{2} \frac{d v}{d z}$ at $\left(x^{\prime} y^{\prime} z^{\prime}\right)$.

Again, if we take a force $-\mathrm{R}$ parallel to $\mathrm{O} z$ at $\left(x^{\prime} y^{\prime} z^{\prime}\right)$ and a force $+\mathrm{R}$ parallel to $\mathrm{O} z$ at $\left(x^{\prime}, y^{\prime}+g, z^{\prime}\right)$, and proceed to the limit as before, keeping $\mathbf{R} g=\frac{1}{2}$,

the displacements will be $-\frac{1}{2} \frac{d u_{3}}{d y},-\frac{1}{2} \frac{d v_{3}}{d y},-\frac{1}{2} \frac{d w_{3}}{d z}$; the resultant of the forces on the element is again a couple of moment $\frac{1}{2}$ in the $y z$ plane;

the work done by these forces acting through $u, v, w$ is $\frac{1}{2}\left(\frac{d w}{d y}\right)^{\prime}$. 
Taking these two doublets together, the displacements are

$$
\begin{aligned}
& u_{4}=\frac{1}{2}\left(\frac{d u_{2}}{d z}-\frac{d u_{3}}{d y}\right)=0 \\
& v_{4}=\frac{1}{2}\left(\frac{d v_{9}}{d z}-\frac{d v_{3}}{d y}\right)=\frac{1}{8 \pi \mu} \frac{d}{d z}\left(\frac{1}{r}\right) \\
& w_{4}=\frac{1}{2}\left(\frac{d w_{2}}{d z}-\frac{d w_{3}}{d y}\right)=-\frac{1}{8 \pi \mu} \frac{d}{d y}\left(\frac{1}{r}\right) ;
\end{aligned}
$$

the resultant of the forces at $\left(x^{\prime} y^{\prime} z^{\prime}\right)$ is a unit couple in the $y z$ plane; the work done on any displacements $u, v, w$ is $\frac{1}{2}\left(\frac{d w}{d y}-\frac{d v}{d z}\right)^{\prime}$ $=\omega_{1}^{\prime}$, the value at $\left(x^{\prime} y^{\prime} z^{\prime}\right)$ of the $x$-rotation in the systen $u, v, w$.

Similar results hold for the similarly derived systems

$$
\begin{array}{rlrl}
u_{5} & =-\frac{1}{8 \pi \mu} \frac{d}{d z}\left(\frac{1}{r}\right) & u_{6} & =\frac{1}{8 \pi \mu} \frac{d}{d y}\left(\frac{1}{r}\right) \\
v_{5}=0 & v_{6}=-\frac{1}{8 \pi \mu} \frac{d}{d x}\left(\frac{1}{r}\right) \\
w_{6}=\frac{1}{8 \pi \mu} \frac{d}{d x}\left(\frac{1}{r}\right) & w_{6}=0
\end{array}
$$

The tractions on the surface $S$ required to maintain these we denote by $\quad \mathrm{F}_{4}, \mathrm{G}_{4}, \mathrm{H}_{4} ; \quad \mathrm{F}_{5}, \mathrm{G}_{5}, \mathrm{H}_{5} ; \quad \mathrm{F}_{6}, \mathrm{G}_{6}, \mathrm{H}_{6}$.

It is to be noticed that the resultant of the tractions $F_{4}, G_{4}, H_{4}$ on $S$ must be a negative unit couple in the $y z$ plane, and similarly with the others.

(b) Take next the following system of applied forces:

$$
\begin{aligned}
& \text { parallel to } \mathrm{O} x,-\mathrm{P} \text { at }\left(x^{\prime} y^{\prime} z^{\prime}\right) \text { and }+\mathrm{P} \text { at }\left(x^{\prime}+f, y^{\prime}, z^{\prime}\right) \text {; } \\
& \text { parallel to } \mathrm{O} y,-\mathbf{Q} \text { at }\left(x^{\prime} y^{\prime} z^{\prime}\right) \text { and }+\mathrm{Q} \text { at }\left(x^{\prime}, y^{\prime}+g, z^{\prime}\right) ; \\
& \text { parallel to } \mathrm{O} z,-\mathrm{R} \text { at }\left(x^{\prime} y^{\prime} z^{\prime}\right) \text { and }+\mathbf{R} \text { at }\left(x^{\prime}, y^{\prime}, z^{\prime}+h\right) .
\end{aligned}
$$

Let $\mathrm{P}, \mathrm{Q}, \mathrm{R}$ increase, and $f, g, h$ diminish indefinitely so that

$$
\mathrm{P} f=\mathrm{Q} g=\mathrm{R} h=1 \text {. }
$$


Then ultimately the displacements are

$$
\begin{aligned}
& u_{7}=-\left(\frac{d u_{1}}{d x}+\frac{d u_{2}}{d y}+\frac{d u_{3}}{d z}\right)=-\frac{1}{4 \pi(\lambda+2 \mu)} \frac{d}{d x}\left(\frac{1}{r}\right) \\
& v_{7}=-\left(\frac{d v_{1}}{d x}+\frac{d v_{2}}{d y}+\frac{d v_{3}}{d z}\right)=-\frac{1}{4 \pi(\lambda+2 \mu)} \frac{d}{d y}\left(\frac{1}{r}\right) \\
& w_{7}=-\left(\frac{d w_{1}}{d x}+\frac{d w_{2}}{d y}+\frac{d w_{3}}{d z}\right)=-\frac{1}{4 \pi(\lambda+2 \mu)} \frac{d}{d z}\left(\frac{1}{r}\right)
\end{aligned}
$$

the applied forces are in equilibrium;

the work done on any displacements $u, v, w$ is $\left(\frac{d u}{d x}+\frac{d v}{d y}+\frac{d w}{d z}\right)^{\prime}$, the value at $\left(x^{\prime} y^{\prime} z^{\prime}\right)$ of the delatation $\triangle$ in the system $u, v, w$. The tractions on $\mathrm{S}$ required to maintain $u_{7}, v_{7}, w_{7}$ we denote by $\mathrm{F}_{7}, \mathrm{G}_{7}, \mathrm{H}_{7}$.

10. Consider now any system of displacements $u, v, w$ of the solid bounded by the surface $S$, produced by surface tractions alone, F, G, H.

Apply Betti's Theorem to this system taken along with each of the above systems $u_{1}, v_{1}, w_{1}$, etc., in turn: we find

$$
\begin{aligned}
& u^{\prime}=\iint\left(\mathrm{F} u+\mathrm{F} v_{1}+\mathrm{H} w_{1}\right) d \mathrm{~S}-\iint\left(\mathrm{F}_{1} u+\mathrm{G}_{1} v+\mathrm{H}_{1} w\right) d \mathrm{~S} \\
& v^{\prime}=\iint\left(\mathrm{F} u_{2}+\mathrm{G} v_{2}+\mathrm{H} w_{2}\right) d \mathrm{~S}-\iint\left(\mathrm{F}_{2} u+\mathrm{G}_{2} v+\mathrm{H}_{2} w\right) d \mathrm{~S} \\
& w^{\prime}= \iint\left(\mathrm{F} u_{3}+\mathrm{G} v_{3}+\mathrm{H} w_{3}\right) d \mathrm{~S}-\iint\left(\mathrm{F}_{3} u+\mathrm{G}_{3} v+\mathrm{H}_{3} w\right) d \mathrm{~S} \\
& \omega_{1}^{\prime}=\iint\left(\mathrm{F} u_{4}+\mathrm{G} v_{4}+\mathrm{H} w_{4}\right) d \mathrm{~S}-\iint\left(\mathrm{F}_{4} u+\mathrm{G}_{4} v+\mathrm{H}_{4} w\right) d \mathrm{~S} \quad \text { with two similar equations }- \\
& \Delta^{\prime}=\iint\left(\mathrm{F} u_{7}+\mathrm{G} v_{7}+\mathrm{H} w_{7}\right) d \mathrm{~S}-\iint\left(\mathrm{F}_{7} u+\mathrm{G}_{7} v+\mathrm{H}_{7} w\right) d \mathrm{~S}
\end{aligned}
$$

The equations from (4) to (7) are those on which Betti founds his method of solving the equations of equilibrium under given surface conditions; we propose to develop a similar method from the more fundamental relations (1), (2), (3). 
It may be noticed that if both surface displacements and surface tractions were given, these relations would give explicitly the values of the displacements at any internal point $\left(x^{\prime} y^{\prime} z^{\prime}\right)$.

11. Suppose, in the first place, that the displacements $u, v, w$ are given at every point of $\mathrm{S}$; it is required to find $u, v, w$ at $\left(x^{\prime} y^{\prime} z^{\prime}\right)$.

Let $F_{1}^{\prime}, G_{1}^{\prime}, H_{1}^{\prime}$ be the tractions on $\mathbf{S}$ required to give surface displacements equal to $u_{1}, v_{1}, w_{1}$, the solid being free from internal applied force; and in this case let $u_{1}^{\prime}, v_{1}^{\prime}, w_{1}^{\prime}$ be the internal displacements.

Applying Betti's theorem to the systems $u, v, w$ and $u_{1}^{\prime}, v_{1}^{\prime}, w_{1}^{\prime}$ we have, since at the surface $u_{1}^{\prime}, v_{1}^{\prime}, w_{1}^{\prime}$ are equal to $u_{1}, v_{1}, w_{1}$

$$
\iint\left(\mathbf{F} u_{1}+\mathrm{G} v_{1}+\mathrm{H} w_{1}\right) d \mathrm{~S}-\iint\left(\mathrm{F}_{1}^{\prime} u+\mathrm{G}_{1}^{\prime} v+\mathrm{H}_{1}^{\prime} w\right) d \mathrm{~S}=0 .
$$

Combining this with (1) of $\S 10$, we get

$$
u^{\prime}=\iint\left\{\left(\mathrm{F}_{1}^{\prime}-\mathrm{F}_{1}\right) u+\left(\mathrm{G}_{1}{ }^{\prime}-\mathrm{G}_{1}\right) v+\left(\mathrm{H}_{1}{ }^{\prime}-\mathrm{H}_{1}\right) w\right\} d \mathrm{~S} .
$$

Hence if $F_{1}^{\prime}, G_{1}^{\prime}, H_{1}^{\prime}$ can be found, the value of $u$ at any internal point $\left(x^{\prime} y^{\prime} z^{\prime}\right)$ is determined.

We observe that the tractions $F_{1}-F_{1}^{\prime}, G_{1}-G_{1}^{\prime}, H_{1}-H_{1}^{\prime}$, acting along with the unit force at $\left(x^{\prime} y^{\prime} z^{\prime}\right)$ give zero displacements at the surface $S$.

Similar relations may at once be written down for $v^{\prime}, w,{ }^{\prime}$ for the rotations, and for the dilatation at $\left(x^{\prime} y^{\prime} z^{\prime}\right)$.

12. The process just explained for solving the problem of given surface displacements, requires us to find surface tractions which, acting along with a given internal source of strain, will hold the surface $f x e d$; the corresponding process, when the surface tractions are given, is more complicated, since the surface cannot be free, unless the internal applied forces are in equilibrium.

For this application we shall therefore suppose applied to the solid, in addition to a unit force at the point whose displacement is to be found, a balancing force and couples acting on the element at some selected point of the solid, taken as origin of coordinates; in a sphere, for example, this point would naturally be taken at the 
centre; in a solid bounded by a plane, or by two parallel planes, it would be convenient to take it at an infinite distance.

Denote by capital letters $U, V, W$ the displacements at any point of the solid arising from a source at the origin, of any of the types already dealt with, and retain suffixes to denote the type of the source. For example, the displacements due to a unit force parallel to $\mathrm{O} x$ applied at the origin denote by $\mathrm{U}_{1} \mathrm{~V}_{1} \mathrm{~W}_{1}$; the displacements due to a source at $\mathrm{O}$ of the $\left(u_{4} v_{4} w_{4}\right)$ type, denote by $\mathrm{U}_{4} \mathrm{~V}_{4} \mathrm{~W}_{4}$.

A unit force parallel to $\mathrm{O} x$ at $\left(x^{\prime} y^{\prime} z^{\prime}\right)$ is balanced by a negative unit force parallel to $\mathrm{O} x$ at $\mathrm{O}$, with a couple $-z^{\prime}$ about $\mathrm{O} y$, and a couple $+y^{\prime}$ about $\mathrm{O} z$.

Hence the displacements

$$
\begin{aligned}
& u_{8}=u_{1}-\mathrm{U}_{1}-z^{\prime} \mathrm{U}_{5}+y^{\prime} \mathrm{U}_{6} \\
& v_{8}=v_{1}-\mathrm{V}_{1}-z^{\prime} \mathrm{V}_{5}+y^{\prime} \mathrm{V}_{6} \\
& w_{8}=w_{1}-\mathrm{W}_{1}-z^{\prime} \mathrm{W}_{5}+y^{\prime} \mathrm{W}_{6}
\end{aligned}
$$

will give rise to tractions $F_{8}, G_{8}, H_{8}$ on the surface, which are in equilibrium.

Apply Petti's Theorem to the systems $u, v, w$ and $u_{8}, v_{R}, w_{\mathrm{R}}$. By the results of $\S 10$, we have

$$
\begin{gathered}
\iint\left(\mathrm{F} u_{8}+\mathrm{G} v_{8}+\mathrm{H} w_{8}\right) d \mathrm{~S}-\iint\left(\mathrm{F}_{8} u+\mathrm{G}_{8} v+\mathrm{H}_{8} w\right) d \mathrm{~S} \\
=u^{\prime}-u-z^{\prime} \omega_{2}+y^{\prime} \omega_{3},
\end{gathered}
$$

where $u^{\prime}$ is the $x$-displacement at $\left(x^{\prime} y^{\prime} z^{\prime}\right)$, and $u, \omega_{2} \omega_{3}$ are the values at $O$ of the $x$-displacement and the $y$ and $z$ rotations, all in the system $u, v, w$.

Let $u_{8}^{\prime}, v_{8}^{\prime}, w_{8}^{\prime}$ be the displacements due to the equilibrating system $F_{8}, G_{B}, H_{8}$ with no internal force.

By Betti's Theorem

$$
\iint\left(\mathrm{F} u_{\mathrm{g}}^{\prime}+\mathrm{G} v_{8}^{\prime}+\mathrm{H} w_{8}^{\prime}\right) d \mathrm{~S}-\iint\left(\mathrm{F}_{8} u+\mathrm{G}_{8} v+\mathrm{H}_{8} v\right) d \mathrm{~S}=0 .
$$

Combining this with (8) we have

$$
u^{\prime}-u-z^{\prime} \omega_{2}+y^{\prime} \omega_{3}=\iint\left\{\mathrm{F}\left(u_{8}-u_{8}^{\prime}\right)+\mathrm{G}\left(v_{8}-v_{8}{ }^{\prime}\right)+\mathrm{H}\left(w_{8}-w_{8}^{\prime}\right)\right\} d \mathrm{~S}
$$

Clearly $u_{8}-u_{8}^{\prime}, v_{8}-v_{8}^{\prime}, w_{8}-w_{8}^{\prime}$ are the displacements due to 
the compound system of sources giving rise in an infinite solid to $u_{8}, v_{8}, w_{8}$, in the case when the surface is free.

The result illustrates the known principle that when the surface tractions are given, the displacements are indeterminate to the extent of an arbitrary displacement of the solid as a rigid body.

The solution can be made determinate if it is arranged that in all cases of given surface tractions, the position of the solid shall be so adjusted that displacements and rotations vanish at some one assigned point.

If this point be the point $O$ above, we have simply

$$
u^{\prime}=\iint\left\{\mathrm{F}\left(u_{8}-u_{8}{ }^{\prime}\right)+\mathrm{G}\left(v_{8}-v_{8}{ }^{\prime}\right)+\mathbf{H}\left(w_{8}-w_{8}{ }^{\prime}\right)\right\} d \mathrm{~S} .
$$

Similar relations hold, of course, for $v^{\prime}, w^{\prime}$.

13. Since the internal force producing the system $u_{4} v_{\downarrow} w_{\downarrow}$ reduces to a unit couple in the $y z$ plane on the element at $\left(x^{\prime} y^{\prime} z^{\prime}\right)$, it follows that the displacements

$$
\begin{aligned}
& u_{9}=u_{4}-\mathrm{U}_{4} \\
& v_{9}=v_{4}-\mathrm{V}_{4} \\
& w_{9}=w_{4}-\mathrm{W}_{4}
\end{aligned}
$$

will require on the surface $S$ tractions $F_{9} G_{9} H_{9}$ which are in equilibrium.

Apply Betti's Theorem to $u, v, w$ and $u_{0} v_{0} w_{\theta}$.

$$
\begin{gathered}
\text { Hence } \iint\left(\mathrm{F} u_{9}+\mathrm{G} v_{0}+\mathrm{H} w_{\theta}\right) d \mathrm{~S}-\iint\left(\mathrm{F}_{9} u+\mathrm{G}_{9} v+\mathrm{H}_{9} w\right) d \mathrm{~S} \\
=\omega_{1}^{\prime}-\omega_{1}
\end{gathered}
$$

$\omega_{1}^{\prime}, \omega_{1}$ being the $x$-rotations in the system $u, v, w$ at $\left(x^{\prime} y^{\prime} z^{\prime}\right)$ and $O$ respectively.

Let $u_{9}^{\prime}, v_{9}^{\prime}, w_{9}^{\prime}$ be the displacements due to the equilibrating tractions $F_{9}, G_{9}, H_{9}$ with no internal force.

Hence

$$
\iint\left(\mathrm{F} u_{9}^{\prime}+\mathrm{G} v_{9}^{\prime}+\mathbf{H} w_{9}^{\prime}\right) d \mathrm{~S}-\iint\left(\mathrm{F}_{9} u+\mathrm{G}_{9} v+\mathrm{H}_{9} w\right) d \mathrm{~S}=0 .
$$

Combining with (9), we have

$$
\omega_{1}^{\prime}-\omega_{1}=\iint\left\{\mathrm{F}\left(u_{9}-u_{9}^{\prime}\right)+\mathrm{G}\left(v_{9}-v_{9}^{\prime}\right)+\mathrm{H}\left(w_{9}-w_{9}^{\prime}\right)\right\} d \mathrm{~S} .
$$

Similarly for the $y$ and $z$ rotations.

These formulae for the rotations, and the corresponding formulae 
for the dilation, may of course be deduced directly from the equations of $\$ \S 11,12$, by differentiation with respect to $\left(x^{\prime} y^{\prime} z^{\prime}\right)$. It may be noted that Betti obtains in place of (9) a formula giving $\omega_{1}^{\prime}$ simply without $\omega_{1}$, but he overlooks the remark at the beginning of $\$ 12$. The error is not corrected in Dr Love's text-book.

14. The general problem of an elastic solid with given bodily forces and given surface tractions or displacements, may be divided into two parts ; in the first, we suppose the bodily force to be null, and the surface tractions or displacements to have their given values; in the second, we suppose the bodily forces to exist, but the surface to be free or fixed. The first part we have dealt with; suppose now that any system of bodily forces $X, Y, Z$ per unit volume exists in the solid, and that

$(a)$ the surface is fixed.

Referring to $\S 11$, the displacements when a unit $x$-force is applied at $\left(x^{\prime} y^{\prime} z^{\prime}\right)$, and the surface is fixed, are

$$
u_{1}-u_{1}^{\prime}, \quad v_{1}-v_{1}^{\prime}, \quad w_{1}-w_{1}^{\prime} .
$$

Take this system with the system $u, v, w$ due to $\mathrm{X}, \mathrm{Y}, \mathrm{Z}$. Betti's Theorem gives for $u^{\prime}$ the $x$-displacement at $\left(x^{\prime} y^{\prime} z^{\prime}\right)$ in the system $u, v, w$.

$$
u^{\prime}=\iiint\left\{\mathrm{X}\left(u_{1}-u_{1}{ }^{\prime}\right)+\mathrm{Y}\left(v_{1}-v_{1}{ }^{\prime}\right)+\mathrm{Z}\left(w_{3}-w_{1}^{\prime}\right)\right\} d x d y d z
$$

the integral being taken over the whole solid.

Similarly with $v,^{\prime} w^{\prime}$.

(b) The surface is free.

Referring to $\$ 12$, the displacements due to a unit $x$-force at $\left(x^{\prime} y^{\prime} z^{\prime}\right)$ with balancing sources at 0 , when the surface is free, are

$$
u_{8}-u_{8}^{\prime}, \quad v_{8}-v_{8}^{\prime}, \quad w_{8}-w_{8}^{\prime} .
$$

Taking this system with the system $u, v, w$ due to $\mathrm{X}, \mathrm{Y}, \mathrm{Z}$ when the surface is free, we have

$u^{\prime}-u-z^{\prime} \omega_{2}+y^{\prime} \omega_{3}=\iiint\left\{\mathrm{X}\left(u_{\mathrm{B}}-u_{8}^{\prime}\right)+\mathrm{Y}\left(v_{8}-v_{\mathrm{s}}{ }^{\prime}\right)+\mathrm{Z}\left(w_{\mathrm{8}}-w_{\mathrm{8}}{ }^{\prime}\right)\right\} d x d y d z$.

When, in the general problem, the systems of bodily force and of surface traction are not separately in equilibrium, a slight modification of the process, such as is exemplified in $\$ 12$, will be required, but this need hardly be dealt with at length. 